\title{
Radiometric Active Indoor Imaging in the W-Band
}

\author{
M. Canavero • A. Murk • C. Mätzler • D. Nötel • \\ Johann Huck
}

Received: 30 May 2013 / Accepted: 10 December 2013/

Published online: 22 December 2013

(C) Springer Science+Business Media New York 2013

\begin{abstract}
Millimeter wave passive imaging systems constitute a good compromise between resolution and penetration depth for a variety of imaging applications. In an outdoor scenario, the cold sky radiation, interacting with the reflectivity characteristics of the targets, constitutes the main source of contrast in the acquired images. In indoor applications such a source is not available, and higher thermal sensitivity is required. Alternatively, one has to provide an artificial illumination to the scene in order to increase its dynamic range. The implementation of an active source for a passive radiometer can, under certain conditions, increase the contrast of the images acquired and add extra information to the measurement. With such a setup, outdoor systems can be used for indoor observations (the absence of cold sky radiation is compensated with active illumination). The subject of our study is to better understand which kind of source and which setup can provide a diffuse illumination over the targets. This topic was investigated by conducting observations of various indoor scenes with two radiometers in the $\mathrm{W}$-Band, using noise and continuous wave $(\mathrm{CW})$ sources as illumination. In this paper we present the results achieved and our conclusions in order to provide an efficient illumination for indoor environment.
\end{abstract}

Keywords Active Illumination · Radiometer · Indoor · Imaging $\cdot$ W-Band

\section{Introduction}

For many decades different radiometers and polarimeters have been built in order to analyze the environment, retrieving useful information to better understand climate and natural

M. Canavero $(\bowtie) \cdot$ A. Murk $\cdot$ C. Mätzler

Institute of Applied Physics, University of Bern, Sidlerstr. 5, 3012 Bern, Switzerland e-mail: marco.canavero@iap.unibe.ch

A. Murk

e-mail: axel.murk@iap.unibe.ch

D. Nötel $\cdot$ J. Huck

Fraunhofer Institute for High Frequency Physics and Radar Techniques FHR, Neuenahrer Str. 20, 53343 Wachtberg, Germany

D. Nötel

e-mail: denis.noetel@fhr.fraunhofer.de 
phenomenon or for security and military purposes. Such instruments operate well in outdoor scenarios, where cold sky radiation provides an excellent source of contrast for the images, which are constituted by a mixture of diffuse and specular reflections, thermal emissivity and physical temperature of targets [1,5]. Moreover, the compromise between atmospheric/ obscurant penetration and spatial resolution at the millimeter frequency band has been a key factor for the popularity of such instruments. In an indoor environment, however, the contrast provided by the sky no longer exists and passive images become more a measure of the thermal emissivity and physical temperature of targets. Considering that under such condition the temperature difference between features in the imaged scene can be small, signal-to-noise ratio and sensitivity of the imagers become the most relevant factors in obtaining good quality images. This situation may require the use of cooled bolometers, in which the noise temperature is very low and the sensitivity is in the order of $0.1 \mathrm{~K}$ [2]. Alternatively one has to provide an external source of illumination in order to increase the dynamic range of the scene $[3,8]$. Commercial security body scanners are a clear example of such as an approach. Their small scale and high scanning speed are suitable for security applications at certain locations such as airports. The drawbacks of this setup are that only scans of person-size targets are possible and that the target must be strategically oriented to obtain recognition, due to the very confined illumination. Previous experiments showed that using monostatic illumination only the reflections from the target surface planes normal to the observing direction of the imager are measured $[3,9]$, in contrast to the diffuse illumination occurring in outdoor passive images [6]. Another interesting research topic is to find the most suitable type of source for illumination in an indoor environment. We tested coherent sources constituted by synthesizers matched with W-Band extensions and incoherent sources like noise diodes [9]. In order to investigate these topics we performed an indoor test campaign in Thun, Switzerland, in June 2012. The aim of the campaign was to study the feasibility of indoor active radiometry focusing on measuring different targets such as camouflage nets, vehicles and people. We obtained active illumination measurements of a variety of test scenes, yielding images with very high levels of contrast and detail in comparison to previous attempts [9] and comparable to outdoor passive imaging. The outcome of our work demonstrates a successful first step towards achieving an active illumination radiometric environment applicable to a variety of fields. In the next sections, we introduce the radiometers involved in the campaign, give a theoretical introduction to active illumination and the typology of illumination sources involved, describe the setups we utilized, and finally, we analyze and comment on the results achieved during our observations.

\section{Imagers Description}

The Scanning Polarimetric Imaging Radiometer (SPIRA) is a fully polarimetric imager in the $90-92 \mathrm{GHz}$ band $[4,7]$. The instrument can measure simultaneously the 4 Stokes parameters for an observed scene using two orthogonally polarized receiver channels and an analog adding correlation network with $2 \mathrm{GHz}$ of bandwidth. The integration time/pixel is $18 \mathrm{~ms}$ while average acquisition time/image and receiver noise temperature are respectively $5 \mathrm{~min}$ and $600 \mathrm{~K}$.

The antenna consists of a dual polarized corrugated feed horn and a $90^{\circ}$ off-axis parabolic reflector with an effective focal length of $70 \mathrm{~cm}$ and a projected aperture of $45 \mathrm{~cm}$. The co and cross-polar antenna pattern of the instrument has been simulated using software package GRASP. The simulations result in a half beam width of about $0.5^{\circ}$, which has been confirmed by far-field antenna measurements with a $91 \mathrm{GHz}$ transmitter. 
The scene is scanned in two dimensions: the off-set parabolic reflector is rotated around the horizontal bore-sight axis of the feed horn (elevation), whereas the antenna and the polarimeter support are rotated around the vertical axis (azimuth). In both axes, stepping motors with a gear mechanism and optical encoder for the absolute referencing are used. Figure 1 gives a schematic overview of the SPIRA polarimeter. It consists of two heterodyne receiver chains, which are both connected to the feed horn through an orthomode transducer (OMT). The OMT has a polarization isolation of more than $30 \mathrm{~dB}$, which corresponds to $3.2 \%$ mixing of the coand the cross-polar signal in the correlator. This effect has to be corrected by the polarimetric calibration of the instrument. In each channel an additional noise signal from a switchable noise diode can be injected through a directional coupler, which is used together with an ambient temperature blackbody calibration target to calibrate the gain and system temperature of the receiver at the end of each antenna scan. A low noise RF amplifier and a high pass filter follow the coupler, selecting the upper sideband of the subharmonic Schottky mixers. They are fed by a common local oscillator $(44 \mathrm{GHz})$ to ensure the phase coherence between the two receiver channels. Optimum pumping of the mixers and fine adjustment of the phase shift between the channels are achieved by variable attenuators and a phase shifter in the local oscillator path. The down-converted signals are amplified by low-noise IF amplifiers and band limited to frequencies between 2 and $4 \mathrm{GHz}$.

Before the campaign, in order to increase the dynamic range of the signal and avoid the saturation of $\mathrm{H}$ and $\mathrm{V}$ channels, extra attenuators were introduced in the IF chain. The six output channels of the analog correlator network are digitally converted and then combined together to reconstruct the four Stokes parameters (I, Q, U, V) [4].

The second instrument was ROTRAD94 (Fig. 2), a fast scanning radiometer developed by Fraunhofer FHR Institute. It has been optimized for a very fast acquisition time. It employs a $61 \mathrm{~cm}$ reflector with a highly integrated receiver array with four channels mounted on its primary focus. The elevation scanning of the system is performed by a continuous rotation of the antenna with a high angular velocity (approximately $150 \mathrm{rpm}$ ). A slower horizontal rotation axis allows the system to scan in azimuth direction. The radiometer only measures the vertical polarization at a center frequency of $92 \mathrm{GHz}$ with a bandwidth of $4 \mathrm{GHz}$, the integration time/pixel is $50 \mu \mathrm{s}$ and an image is acquired in approximately 30s. Due to the fact

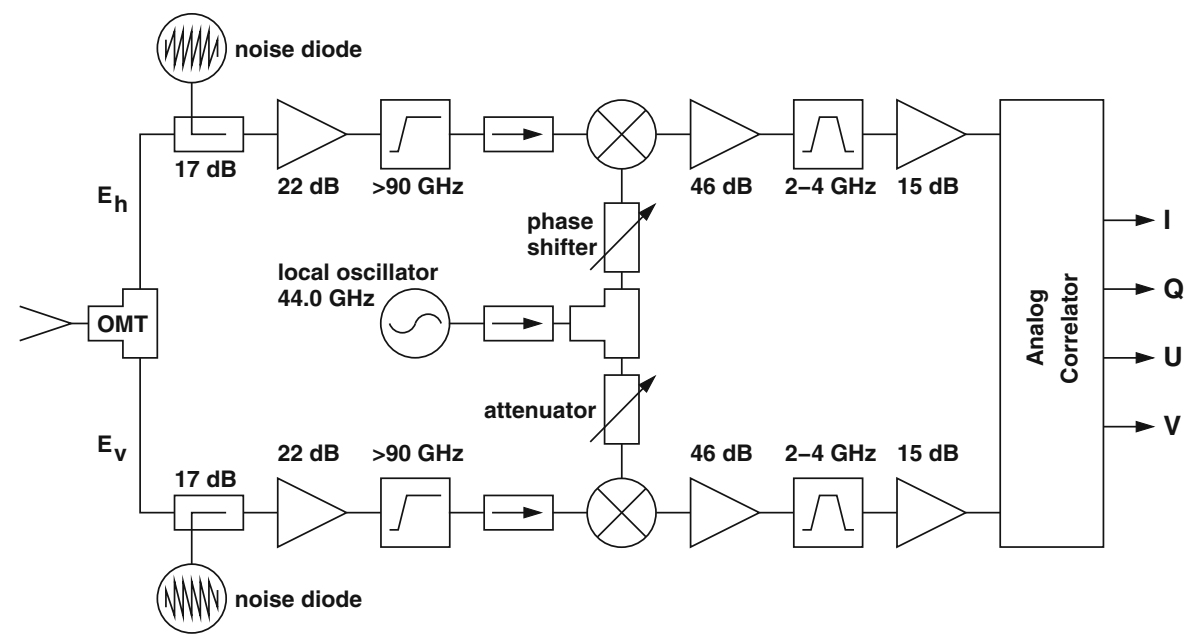

Fig. 1 Block diagram of SPIRA receiver 


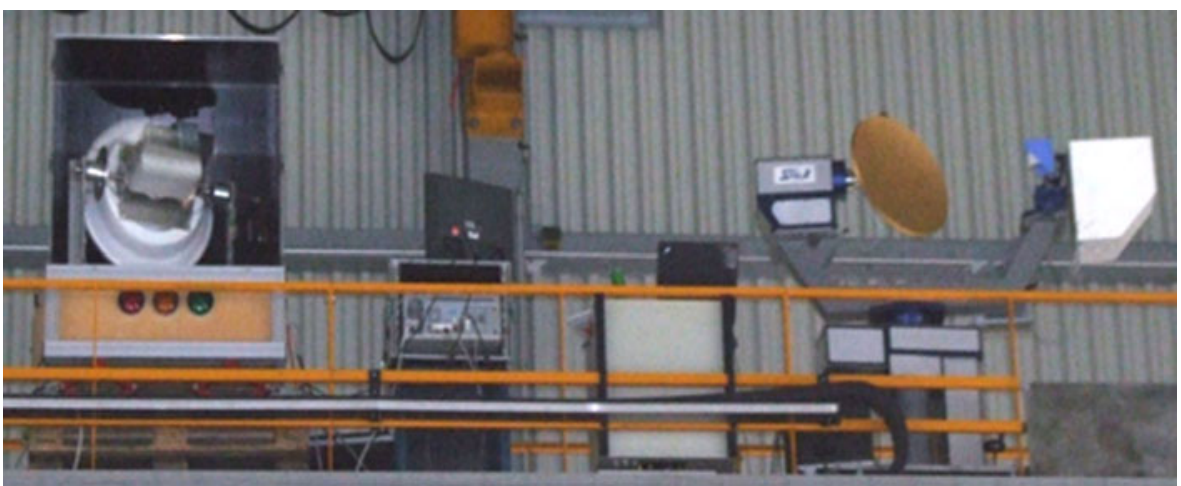

Fig. 2 Rotrad94 (left) and SPIRA (right) during the measuring campaing in 2012

that the instrument is not calibrated, all the images taken by ROTRAD represent voltage values [6]. The characteristics of the two instruments are complementary. While the fast scanning ability of ROTRAD94 has been very useful to quickly scan the scene in order to investigate the effectiveness of each illumination setup, SPIRA provided better quality images in term of thermal sensitivity and was also able to obtain good passive images of the scenes.

\section{Static Illumination and Speckle Noise}

The general relationship between the antenna temperature $T_{a}$ and the received power $P$ for a radiometer with a receiver bandwidth $B$ (in $\mathrm{Hz}$ ) is the following:

$$
P=k T_{a} B
$$

Where $\mathrm{k}=1.38 \cdot 10^{-23} \mathrm{~J} / \mathrm{K}$ is the Boltzmann constant. The minimum variation of temperature measured by the radiometer (sensitivity) is:

$$
\Delta T_{\min }=\frac{T_{a}+T_{n}}{\sqrt{B \tau}}
$$

Where $T_{n}$ is the noise temperature of the receiver and $\tau$ is the integration time of the measurement. Using high receiving bandwidth ( $B=2 \mathrm{GHz}$ for SPIRA) is an advantage not only for the sensitivity of the instrument but also for reducing the speckle noise, as we will discuss later. In order to provide illumination for a scene from a point 1 , at a distance $R_{1}$ from the target and directions $\vartheta_{1}, \varphi_{1}$, and measure the incoming power at the receiver of our radiometer in the point 2 , we have to consider not only the radiation coming from a single scatterer at a distance $R_{2}$ and direction $\vartheta_{2}, \varphi_{2}$ from the receiver, but from every object which contributes to the overall scattered radiation. Starting from the bistatic radar equation:

$$
P_{2}=\frac{P_{1} \lambda^{2} G_{1}\left(\vartheta_{1}, \varphi_{1}\right) G_{2}\left(\vartheta_{2}, \varphi_{2}\right)}{(4 \pi)^{3}} \sigma_{b i}{ }^{2} R_{2}^{2}
$$

Where $G_{1}, G_{2}$ are the gain functions of transmitting and receiving antennas at the given directions, $\lambda$ is the wavelength, $R_{1}$ and $R_{2}$ are respectively the distance between illuminator and scatterer and between the scatterer and receiving antennas, $\sigma_{b i}$ is the scattering crosssection of the target. With the assumption of incoherent superposition to a volume integral (no 
phase relationship between the single scatterers), we can estimate the received power $P_{2}$ from the scattered radiation (defined between a minimum distance $R_{\min }$ to a maximum distance $\left.R_{\max }\right)$ as:

$$
P_{2}=\frac{P_{1} \lambda^{2}}{(4 \pi)^{3}} \int_{R_{\min }}^{R_{\max }} \iint_{4 \pi} \frac{G_{1}\left(\vartheta_{1}, \varphi_{1}\right) G_{2}\left(\vartheta_{2}, \varphi_{2}\right)}{R_{1}{ }^{2}} \gamma_{b i} d \Omega_{2} d R_{2}
$$

where $\gamma_{b i}$ is the bistatic volume scattering coefficient, $\gamma_{b i}=\Sigma \sigma_{b i}\left(R_{2}\right) N\left(R_{2}\right)$. Where $\sigma_{b i}$ is the bistatic cross section of each scatterer [16] and $\mathrm{N}\left(\mathrm{R}_{2}\right)$ is their particle number size distribution. The volume element is represented by $d V=d R_{2} R_{2}^{2} d \Omega_{2}$ and $d \Omega_{2}=d \vartheta_{2} d \varphi_{2}$. The incoherent sum of the power is justified by the fact that averaging over coeherent speckle converges towards $\mathrm{P}_{2}$ in Eq. 4. The latter equation can be simplified assuming the target as a single isotropic scatterer $[8,14]$. In this case it is possible to use the normalized scattering cross section $\sigma_{n}=\sigma_{b i} / A$, where $A$ is the illuminated surface. The surface $d A$ is also seen from the observer under the solid angle $d \Omega_{2}=d A \cos \phi / R_{2}{ }^{2}$ and is related to the antenna aperture, while $\phi$ is the angle between the surface normal and the direction to the receiver. Thus the integral over the solid angle $\Omega$ of the scene is:

$$
P_{2}=\frac{P_{1} \lambda^{2}}{(4 \pi)^{3}} \iint_{\Omega} \frac{G_{1}\left(\vartheta_{1}, \varphi_{1}\right) G_{2}\left(\vartheta_{2}, \varphi_{2}\right)}{R_{1}^{2} \cos \phi} \sigma_{n} d \Omega_{2}
$$

Where $\Omega=4 \pi$. If we then consider a monostatic illumination [14], in which transmitting and receiving antenna are placed in the same position $\left(R_{1}=R_{2}=R\right)$ and $G_{1}=G_{2}=G$, the received power can be written as $[8,17]$ :

$$
P_{2}=\frac{P_{1} \lambda^{2} \sigma^{0} K G_{\max }}{(4 \pi)^{2} R^{2} \cos \phi}=\frac{P_{1} \sigma^{0} K A_{e f f, \max }}{4 \pi R^{2} \cos \phi}
$$

Where $\sigma^{0}$ is the backscatter coefficient, $K$ is a factor relative to the antenna characteristic ( $K=0.5$ for Gaussian beam, $K=0.25$ for standard gain horn [17]), $G_{\max }$ is the maximum antenna gain (sidelobes contributions are neglected) and the antenna effective area $A_{\text {eff,max }}$ is given by the relationship:

$$
A_{e f f, \max }=\frac{\lambda^{2} G_{\max }}{4 \pi}
$$

Eq. 6 can be then used to estimate the received power using simple geometrical and radiometrical parameters, such the backscattering coefficient. For reflecting surfaces, $\sigma^{0}$ shows a strong angular dependency, and can be generally expressed in the form [5]:

$$
\sigma^{0}=s(\cos \phi)^{\alpha}
$$

Considering a Lambertian scatterer (ideal diffusely reflecting surface) $\alpha=2, \mathrm{~s}=4 \mathrm{r}$, where $r$ is the reflectivity of the surface. Combining Eq. 6 and 8 we can calculate the active radiometric receiving power from a simple geometry target as:

$$
P_{2}=r \cos \phi \frac{P_{1} K A_{e f f, \text { max }}}{\pi R^{2}}=P_{1} K r \cos \phi\left(\frac{a_{e f f}}{R}\right)^{2}
$$

$a_{\text {eff }}$ is the effective antenna radius. In our experimental setup, receiver and transmitter are not in the same position as discussed in section 9 (see the scheme in Fig. 27), and their propagation directions formed a $90^{\circ}$ angle. The received power $P_{2}$ can be then written as: 


$$
P_{2}=2 P_{1} K r \cos \phi \sin \phi\left(\frac{a_{e f f}}{R}\right)^{2}=P_{1} K r \sin (2 \phi)\left(\frac{a_{e f f}}{R}\right)^{2}
$$

Eq. 9 and 10 clearly show the strong angular diversity of the incoming receiving power for a monostatic setup. The receiving power, in fact, is strongly dependent by the $\phi$ angle between the target surface normal and the receiver scan direction. On the other hand, the main advantage of such a setup consists in the relatively low transmitting power necessary to illuminate the scene. As a quantitative example we consider $r=0.1, \mathrm{~K}=0.5, \cos \phi=0.7$, $R=10 \mathrm{~m}$ and $a_{\text {eff }}=0.2 \mathrm{~m}$ (SPIRA antenna). From Eq. 9 we obtain that:

$$
P_{2} / P_{1}=1.4 \cdot 10^{-4}
$$

In order to rise the receiving temperature by $100 \mathrm{~K}$ we have to provide:

$$
T_{1}=100 \cdot P_{1} / P_{2}=7 \cdot 10^{5} \mathrm{~K}
$$

Using now Eq. 1, we obtain a transmitting power $P_{1}=20 \mathrm{nW}$ for a bandwidth $B=2 \mathrm{GHz}$.

Even if this setup seems favorable from the transmitting power point of view, it is impossible to avoid the direct cross talk between transmitter and receiver in a monostatic setup. In order to reduce cross talk, transmitter and receiver have to be separated, resulting in a wider area to be illuminated and a higher transmitting power required in order to uniformly illuminating the scene. Generally the assertion that to illuminate an area of $\mathrm{N}$ times $\mathrm{N}$ pixels, the required increased power is on factor $\mathrm{N}^{2}$ compared to the monostatic geometry is valid. In our example, for a 100x100 pixel image, the required power rises then to $0.2 \mathrm{~mW}$. A similar problem arises if one wants to illuminate the target with full angular diversity using an imaging system with a collecting aperture $D$ and a distance to the target $R$, the power multiplication factor $N_{D}$ has to be considered [3], where:

$$
N_{D} \cong\left(\frac{R}{D}\right)^{2}
$$

In our case $(R=10 \mathrm{~m}, D=0.45 \mathrm{~m})$ we obtain $N_{D} \approx 500$. As we have already mentioned, the previous formulation is intended in an ideal case, where all the realizations of multiple scatterers are averaged and tend to reach the expected values. In the real case, the waves, reflected by the scatterers, are superimposed at the receiver (with different phases), generating interferences. The complex signal at the receiver is in the form $U_{2}=A_{2} e^{(i k z)}$, where $k=2 \pi f$ is the wave number, $\mathrm{z}$ is the wave path, and is composed by the sum of the single reflections from all the $\mathrm{N}$ scatterers in the form:

$$
U_{2}=\sum_{j=1, N} a_{j} \exp \left(i \psi_{j}\right)
$$

With $\psi=k z$. The phase difference of the signal from two scatterers is frequency dependent in the form:

$$
\psi_{i j}=2 \pi f\left(z_{i}-z_{j}\right) / c
$$

Changing the frequency $f$ with the bandwidth $B$, phase dispersion arises:

$$
\Delta \psi_{i j}=2 \pi B\left(z_{i}-z_{j}\right) / c
$$


until reaching a completely decorrelated situation when $\Delta \phi=2 \pi$ for:

$$
z_{i}-z_{j}=\Delta z=c / B
$$

Defining the coherence length as:

$$
L_{c}=\frac{c}{B}
$$

Which represent the path length within which the wave maintains a certain degree of coherence and therefore interference occurs, $\mathrm{c}$ is the speed of light, $B$ is the minimum between receiver and transmitter bandwidth. In the case of visible light, the coherence length is very short and no interference occurs.

In the case of SPIRA, with $B=2 \mathrm{GHz}, L_{c}=15 \mathrm{~cm}$. Then, speckle noise effect is visible in the images. The use of CW sources, with their narrow bandwidth, leads to an increase of visible speckle noise due to the wider coherence length. In order to reduce consistently the speckles, one has to either perform a new measurement (or realization) of the same scene shifting the illuminator of a distance $D$ equal to the diameter of the receiving antenna [8], use broadband incoherent sources like noise diodes or increase the number of emitters creating a planar illumination similar to the cold sky illumination.

\section{Continuous Wave and Noise Sources for Active Illumination}

Regarding the nature of signal for illumination, the Continuous Wave sources (CW) constituted the first option to increase contrast in radiometric images for our indoor environment. In this configuration one or more narrow band coherent signals (within the receiver band) are used to illuminate a target. As already mentioned in this document and in our previous studies $[9,10]$, multiple reflections of coherent sources are the cause of speckle noise in radiometric images. In order to reduce this effect and to achieve a planar illumination, multiple $\mathrm{CW}$ sources are suitably a better choice. Theoretically a frequency sweep is also indicated in order to reduce speckles. If the frequency sweep is set within the single pixel acquisition time of the radiometer, reflections, at different frequencies along the entire receiver bandwidth, could occur at different angles (due to dispersion phenomena) and potentially averages over constructive and destructive interferences. On the other hand CW sources have the advantage that they will provide higher sensitivity and dynamic range, especially when used in combination with digital signal acquisition techniques. When the CW signal source is tuned outside of the IF bandwidth of the passive receiver, it can still be detected with separate narrow band detectors tuned to this external frequency, which would allow observing active and passive images simultaneously.

Noise active illumination was the second possible choice in order to increase contrast and detail in indoor measurements. From previous experiments we conducted, such technique has fewer drawbacks compared to $\mathrm{CW}$ illumination [9, 10]. Speckle noise in active noise images is low due to the statistical characteristics of the signal and the reduced coherence length (see previous section), while saturation of the receiver, due to the fact that the received signal occupies the entire bandwidth, can occur. This problem, however, can be easily handled by tuning the illumination power introducing variable waveguide attenuators.

Concerning the optics of the active illumination system, different rectangular horn antennas have been considered. To cover images with a wide field of view we used open waveguides in 
order to broader the radiation pattern. This approach requires a higher transmitting power, but the angular dependency of the results should be consistently reduced. Using multiple indirect reflections, uncorrelating the transmitting signal also helps to reduce the speckle noise effect. The direct crosstalk between transmitting horns and receiver has to be minimized in such a configuration, and microwave absorbers have been used to minimize the direct view of the sources from the receiver.

\section{Experiment Description}

The main efforts of our experiment have been focused on:

- Testing CW and Noise sources;

- Optimization of the total transmitted power and the frequency range (for CW signals);

- Optimization of the disposition of the sources related to the measured target;

- Define test targets with simple geometry.

The indoor measurement campaign was conducted in the millimeter wave measurement hall of armasuisse (Fig. 3), in Thun, which is usually used for radar cross section measurements of vehicles (a metalized rotating platform is installed on the floor for such a purpose). The walls and the parabolic roof are made of corrugated metal plates. We used such features of the hangar to generate multiple reflections on the metal surfaces, confining at the same time the radiation within the building. The sources have been pointed to the parabolic roof, in order to distribute the radiation homogenously on the rotating platform, which served as our target area, avoiding direct view illumination. The windows on the walls allow a portion of the cold sky radiation to penetrate inside the hall, making the acquisition of passive indoor images possible, when clear sky condition occurred. The SPIRA and Rotrad94 radiometers were placed on an elevated platform at 12 meters above the floor (Fig. 2).

As mentioned above, one of the main goals of our experiment was to compare the performances of $\mathrm{CW}$ versus noise illumination. In order to do so we tested different sources such as noise diodes, synthesizers matched with W-Band modules, high power noise sources $(100 \mathrm{~mW})$, in different configurations. We used convex spherical mirrors and diffuser boxes, in order to maximize the illuminated area. These boxes, built by FHR, are carton boxes internally covered by wrinkled aluminum foils which act as random scattering surfaces. The aperture of

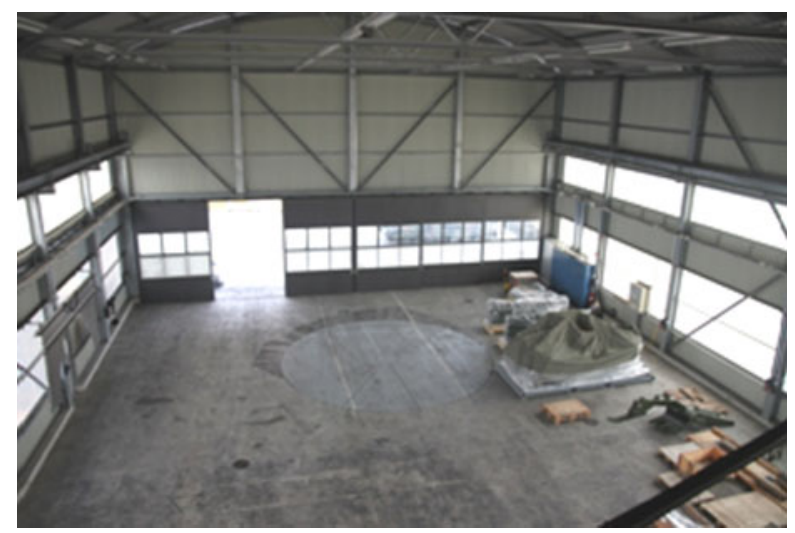

Fig. 3 Internal view of the measurement hall, from the point of view of SPIRA 
the boxes can be covered by a metal grid to increase their scattering characteristic. One of these diffusers and its temperature distribution is shown in Fig. 4 and 5. Along with these components we had also flexible waveguides ( $45 \mathrm{~cm}$ long, $3 \mathrm{~dB}$ of attenuation for each section), corrugated horns (high directivity), broad beam horns, couplers and power dividers.

Different illumination schemes for each scene were tested, focusing on correctly illuminating the platform on which we positioned the targets. Optimization of the various source configurations was time consuming, owing to the fact that this was the first implementation of the instruments in an environment which was spatially large and composed almost entirely of metal walls. At the same time we tested the two techniques for illumination of the scene (CW and noise sources) and comparing those using common targets.

Throughout the experiment, we restricted our range of investigation to the most promising configurations. During this optimization phase the attributes of ROTRAD were crucial. With its fast scanning ability, we were able to quickly scan each source configuration, and subsequently selecting the efficient ones.

\section{Passive and CW Illumination Results}

The presence of windows along the walls of the MMW Hall allowed us to obtain some passive images of the hall due to the partial penetration of cold sky radiation inside the hangar. This was possible, however, only under clear sky conditions. In overcast conditions, the observed passive images showed poor contrast, as expected. The following passive images (Figs. 6 and 7) show how the sensitivity of the instruments is the most important factor in indoor passive radiometry.

SPIRA, due to its continuous calibration scheme after each elevation scan and its lower noise temperature, was the only imager able to provide a discrete contrast in the passive images, while the higher receiver noise in ROTRAD (uncalibrated) lower its SNR. The same scene was then illuminated using a single $\mathrm{CW}$ illuminator ( $20 \mathrm{dBm}$ of transmitted power) placed in front of the platform, pointing at the roof above. An open waveguide was used in

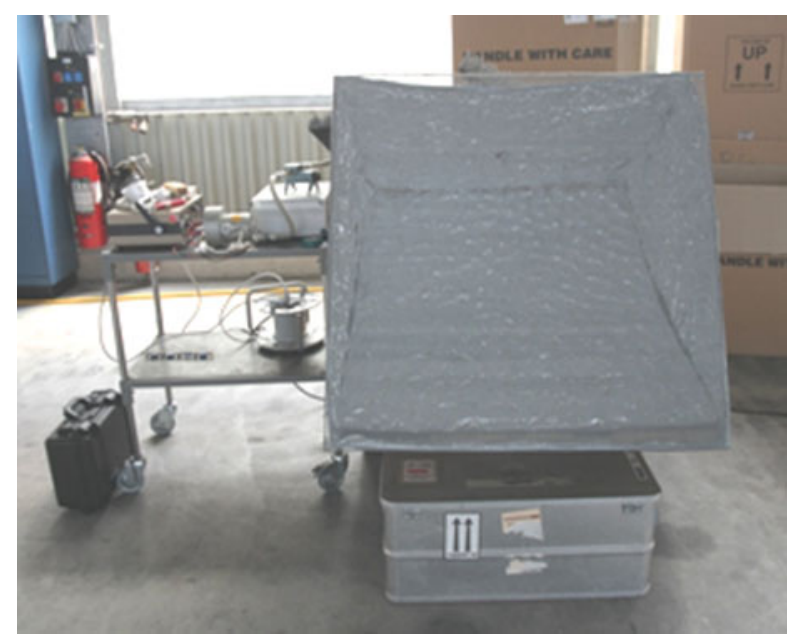

Fig. 4 High power noise source (left) coupled with large size diffuser box (right) 


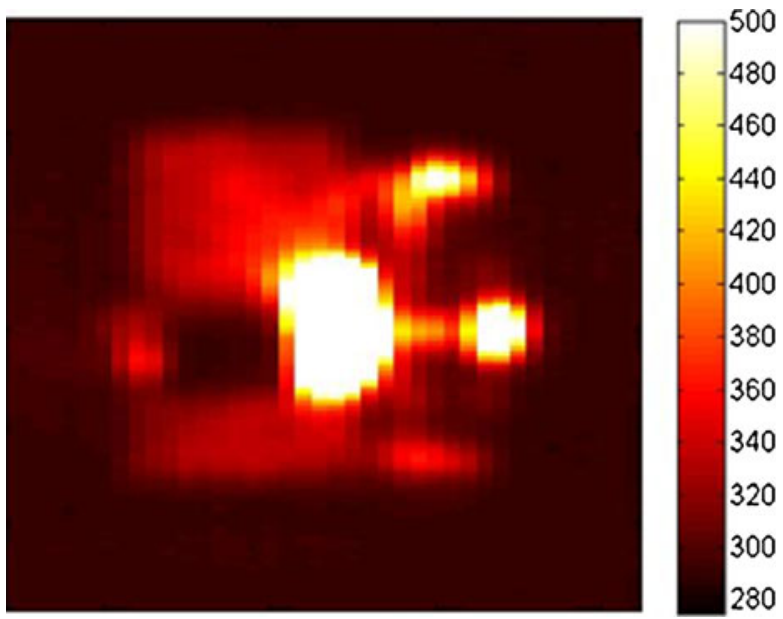

Fig. 5 Power distribution inside the diffuser box of Fig. 4, represented here in thermal scale [K]

order to broaden the radiation pattern as much as possible. As expected for a narrowband source, the speckle noise is dominant (Fig. 8).

A small percentage of the illumination is reflected above the platform. In order to lower this effect, a test scene (Fig. 9), where a car has been parked over the rotating platform, has been illuminatted by pointing two $\mathrm{CW}$ sources the at the upper left and right corner of the hangar. We obtained very noisy images, shown in Figs. 10 and 11.

The vehicle is illuminated, but only a small portion of this radiation is directed backward to the radiometer and two hot circular spots are visible on the hangar gate behind (upper right quadrant of Fig. 10), while speckle noise is dominant over the car and on the surrounding floor, especially on the high reflective rotating platform. The latter of the two pictures (Fig. 11) was obtained redirecting first the illumination over randomly oriented reflecting surfaces in order to decorrelate and diffuse as much as possible the transmitted signal.

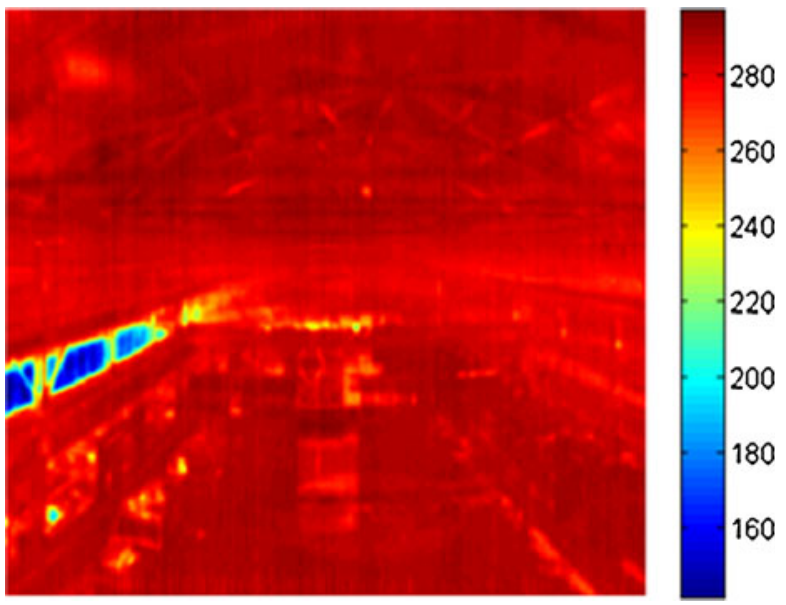

Fig. 6 SPIRA passive image of the hall, in brightness temperature [K] 


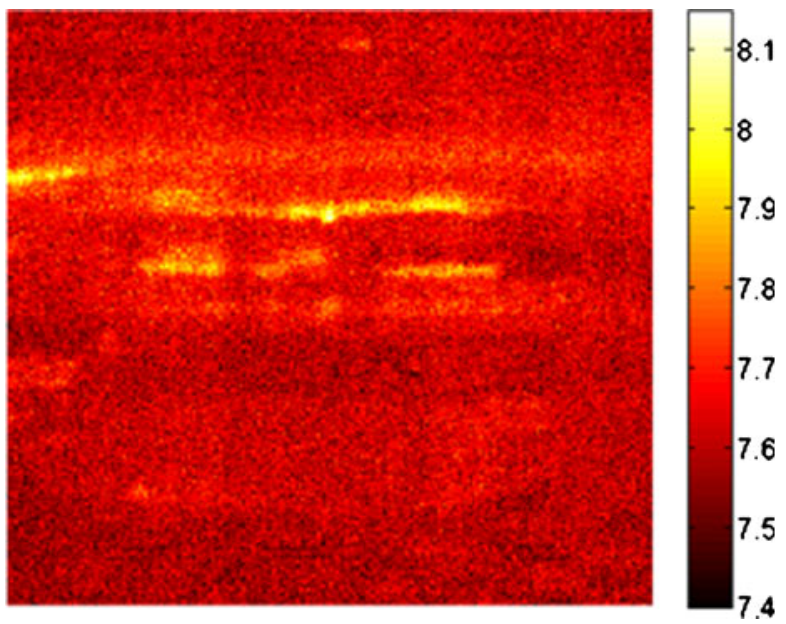

Fig. 7 Rotrad94 passive image of the hall, in voltage scale [V]

We obtained a better distributed illumination over the entire scene, with more predominance on the left side of the target. The platform had more contrast than in the previous image (Fig. 10). The metal top and the front of the car highly reflected the radiation, resulting in a very bright area.

The speckle noise, however, was still dominant and widespread among the image. These results, obtained illuminating the targets with $\mathrm{CW}$ sources, proved that such illumination technique is problematic to handle. Using multiple sources and scattering the radiation only partially may solve the speckle noise problem. In fact, it is very difficult to provide uniform illumination over the entire scene, due to the fact that coherent radiation can only be reflected by the targets (under illumination) through specific angles.
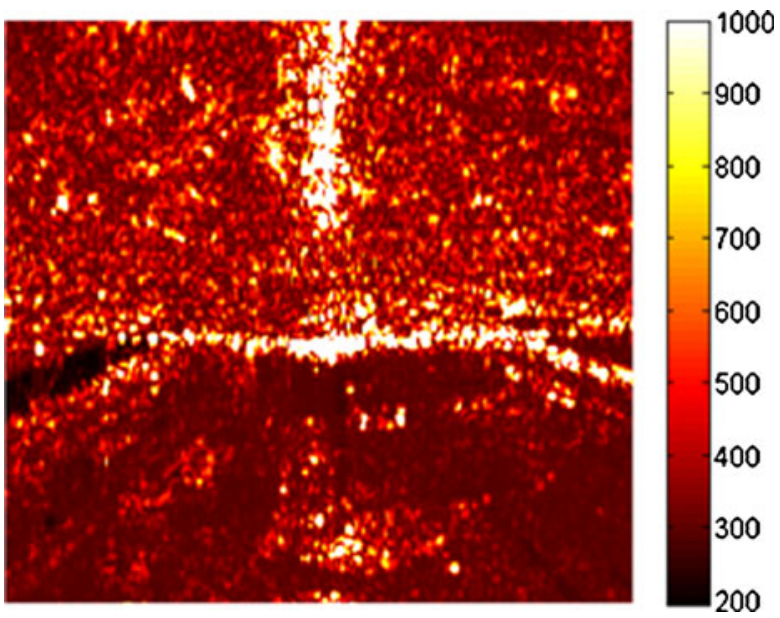

Fig. $8 \mathrm{CW}$ active image from SPIRA in brightness temperature [K]. The scene is the same as Fig. 6. $20 \mathrm{dBm}$ of transmitted power, open waveguide, $91 \mathrm{GHz}$. Speckle noise is dominant 


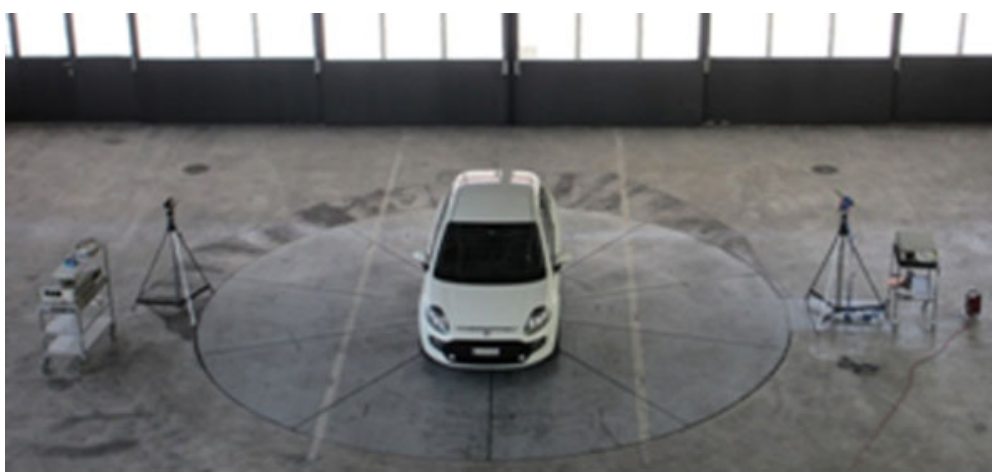

Fig. 9 Optical picture of the car test scene

\section{Noise Illumination Results}

Noise active illumination was the second possible choice in order to increase contrast and detail in indoor measurements. The high power noise source proved to be the best source at our disposal, even if it suffered of power leaking from the source circuits. The amount of transmitted power was far more than necessary to illuminate the hall and extra attenuation stages had to be introduced. Once the source power was correctly tuned, we obtained the first indoor active images that displayed the scene with a satisfactory amount of contrast and detail. In order to obtain better information about the impact of indoor active illumination we arranged the test in Fig. 12, introducing a metal box and absorbers. As expected from theoretical studies, noise illumination tends to saturate the scene but there are no visible speckle effects. While using CW sources, the speckle noise cannot be reduced by redirecting the illumination on randomly oriented surfaces or by using more independent sources (we had two $\mathrm{CW}$ sources at our disposal during the campaign), noise illumination only requires the tuning of the transmitting power in order to maintain the received signal within the dynamic

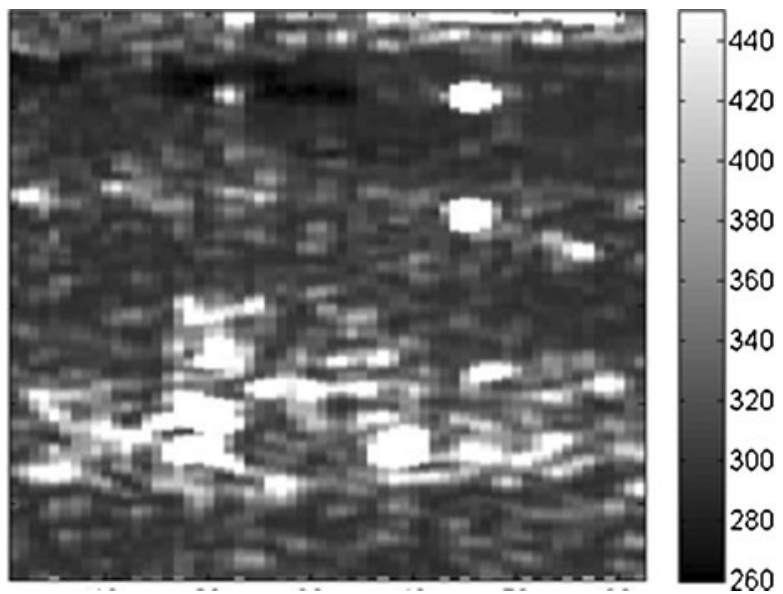

Fig. 10 Active CW image in brightness temperature [K], dual illumination (90.5 and 91.5 GHz), $20 \mathrm{dBm}$ of power 


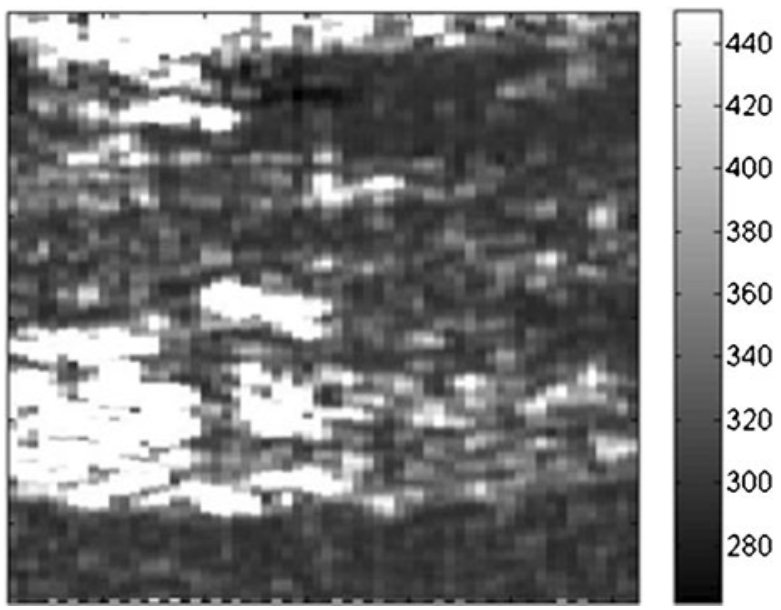

Fig. 11 Active CW image in brightness temperature $[\mathrm{K}]$, dual illumination $(90.5-91.5 \mathrm{GHz})$ through randomly oriented surfaces, $20 \mathrm{dBm}$ of power

range of the receiver. The absorber surfaces over the platform in the passive image (Fig. 13) are expected to be the warmest objects in the scene, while the opposite occurs in the active images. In passive observations the absorbers have the highest brightness temperature (white) over the platform $(294 \mathrm{~K})$. In the active images their temperature increases to $313 \mathrm{~K}$ (dark red), while the adjacent part of the platform rises from approximately $288 \mathrm{~K}$ to $394 \mathrm{~K}$.

The measurements presented here validate our expectations about different effects of application of active noise source illumination above objects with absorbing or reflecting characteristics, enhancing the contrast between them (Fig. 14). Data analysis on the cuts of active radiometric images (i.e. vertical cut of metal box covered by different camouflage nets, Fig. 15) shows a considerably higher correlation factor between images of the same target, covered by two different types of camouflage nets, when noise source are used, compared to CW sources.

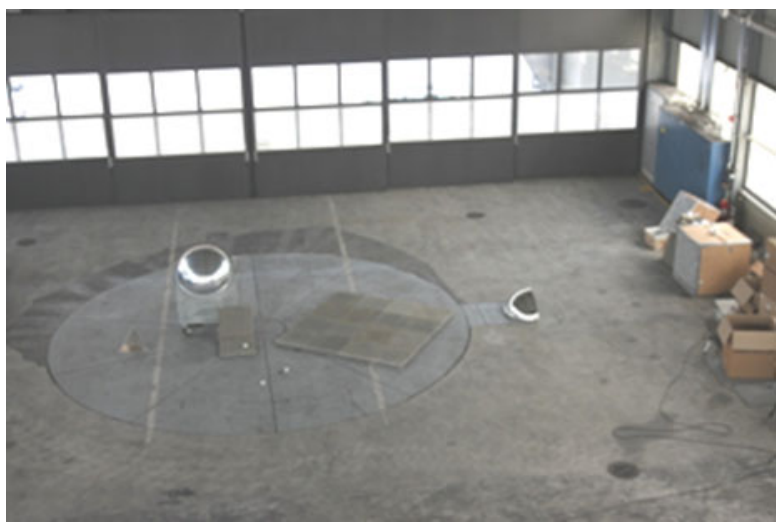

Fig. 12 Test scene for active noise illumination. Metal box and absorbers on the platforms, diffuser box and source on the right 


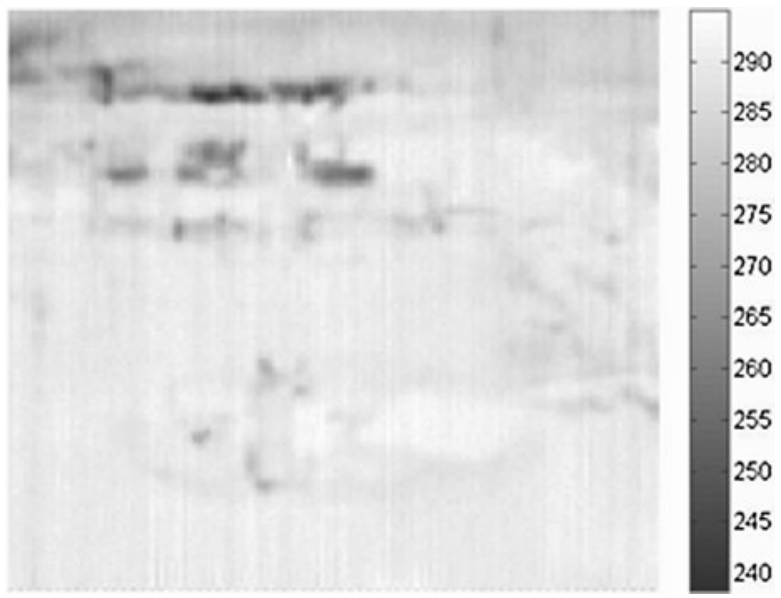

Fig. 13 Passive image of the test scene in Fig. 12, in brightness temperature [K]. Absorbers on the platform at $292 \mathrm{~K}$

We obtained the following correlation values between the same vehicle cuts, when the vehicle is covered by two different camouflage nets and illuminated by noise or CW source:

$$
\begin{aligned}
& \rho_{\text {noise }}=0.96 \\
& \rho_{C W}=0.37
\end{aligned}
$$

As expected, this result confirms that the random reflections, introduced by camouflage nets covering the target, mostly affects the quality of $\mathrm{CW}$ images, lowering the correlation between different images of the same target when it is covered by random scattering surfaces. Different noise images of the same target, instead, present a higher correlation. The latter illumination system is then more robust to random reflections effects which can alter the active image quality.

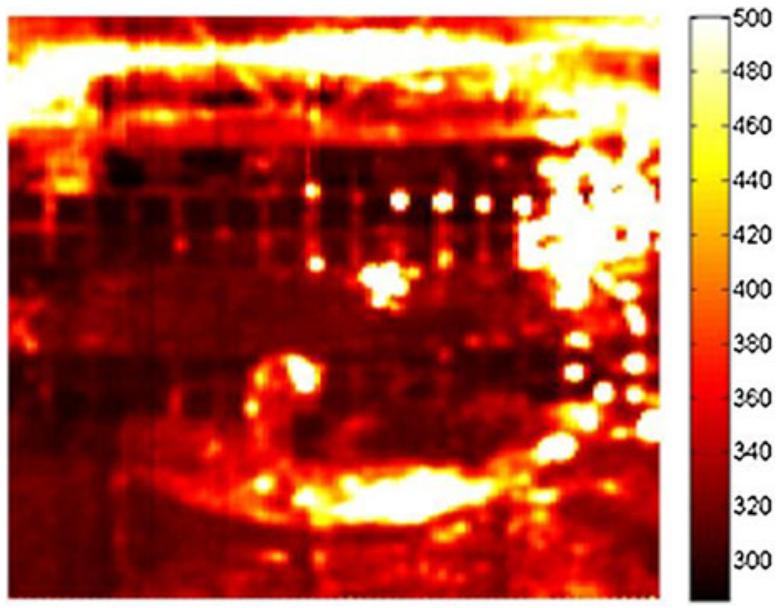

Fig. 14 Active noise illumination image of the test scene in Fig. 12 in brightness temperature [K]. Absorbers on the platform at $313 \mathrm{~K}$ 


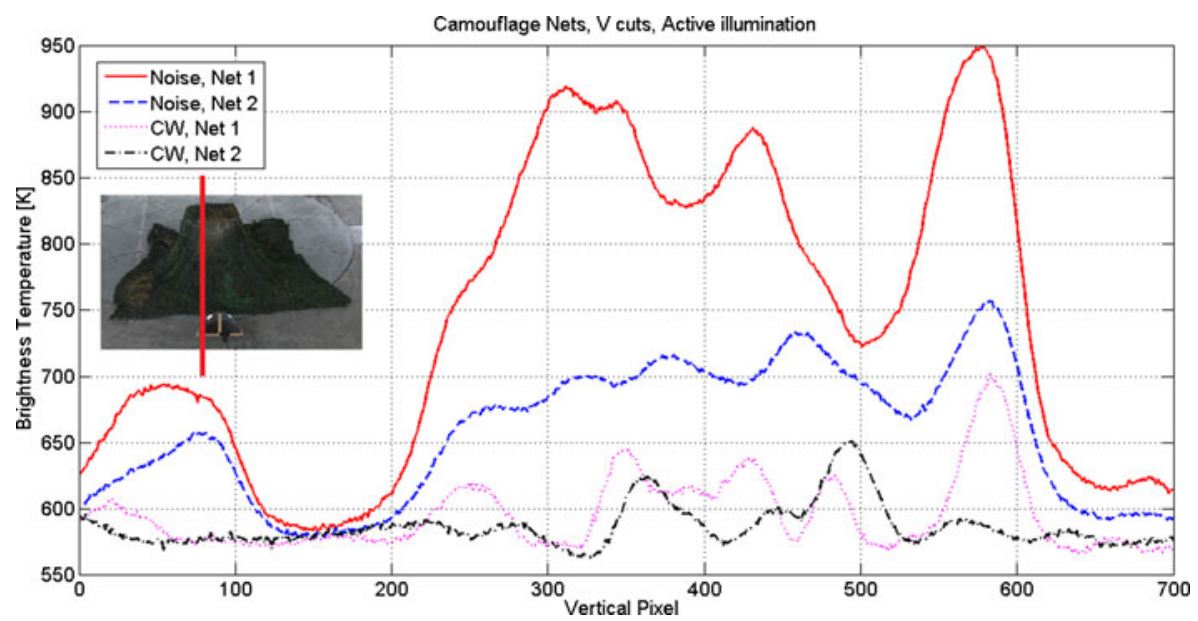

Fig. 15 Vertical cuts (red line in the optical picture) of CW and noise images of the camouflaged box

As we mentioned before, analyzing the differences between passive and active indoor imaging, the sensitivity of the instrument is the most relevant factor in order to obtain a decent SNR for the data when the illumination is switched off, while the dielectric properties of the different components of the target are enhanced under illumination. This improved SNR is clearly visible comparing the data in the passive images of ROTRAD and SPIRA (the latter has higher sensitivity) in Figs. 6 and 7. By introducing noise illumination in the scene, the dynamic range of the images rises due to the increasing reflected radiation from metal parts of the targets. As an example see Fig. 16, where the single components of the vehicle are clearly distinguishable in the active illumination vertical cut (along the red line).

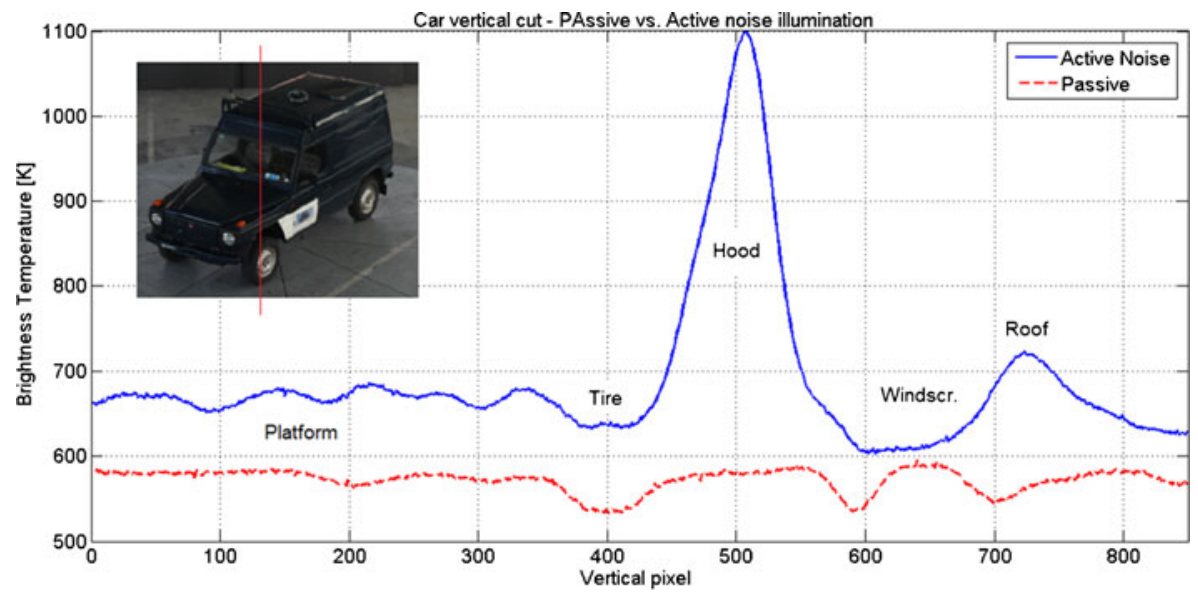

Fig. 16 Vertical cut of passive and active noise images of a Puch. The cut of active illumination image reveals a higher contrast between the different components of the car 


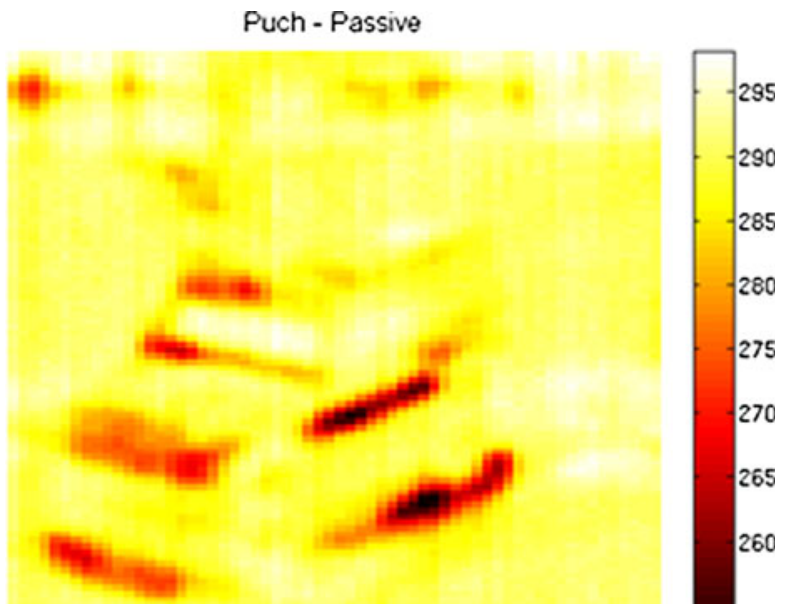

Fig. 17 Passive image from SPIRA of the Puch vehicle, in brightness temperature [K]

\section{Analysis of Power Spectral Density of the Images}

Further analysis on effects of active illumination has been done by calculating the power spectral density (PSD) of each image through a 2 dimensional Fourier transformation. The PSD of an image represents the power distributed over different frequencies of intensity variations. It is widely use in laser applications to characterize the speckle generated by pulsed sources [11] or for surface morphology analysis [12].

The central point of the PSD at coordinates $(0,0)$ represents the continuous power component along the whole image and is generally referred as background power or DC term. It is used to estimate the overall average power of the image. This characteristic is suitable to relate the average amount of power to the relative angle between the target and the

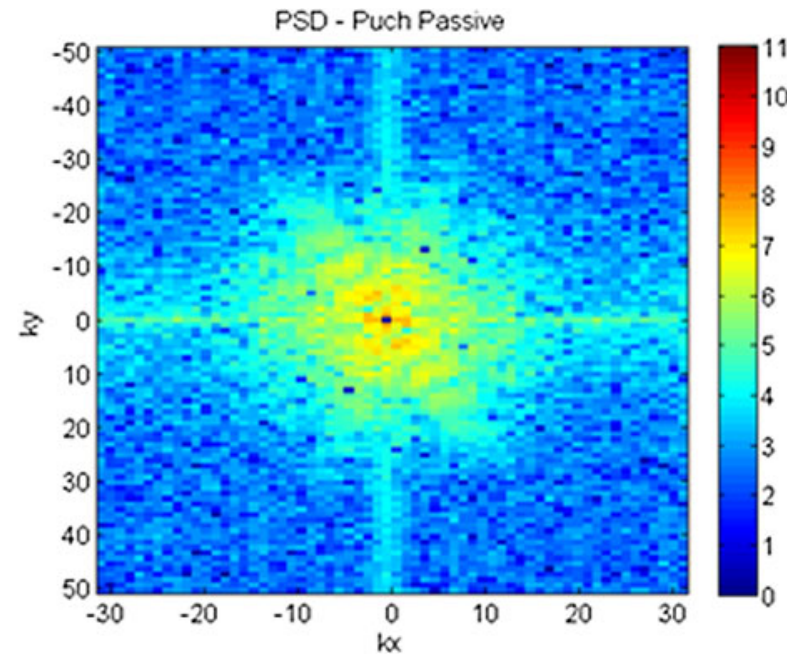

Fig. 18 PSD of the passive image of the Puch (Fig. 17) in logarithmic scale [dB] 


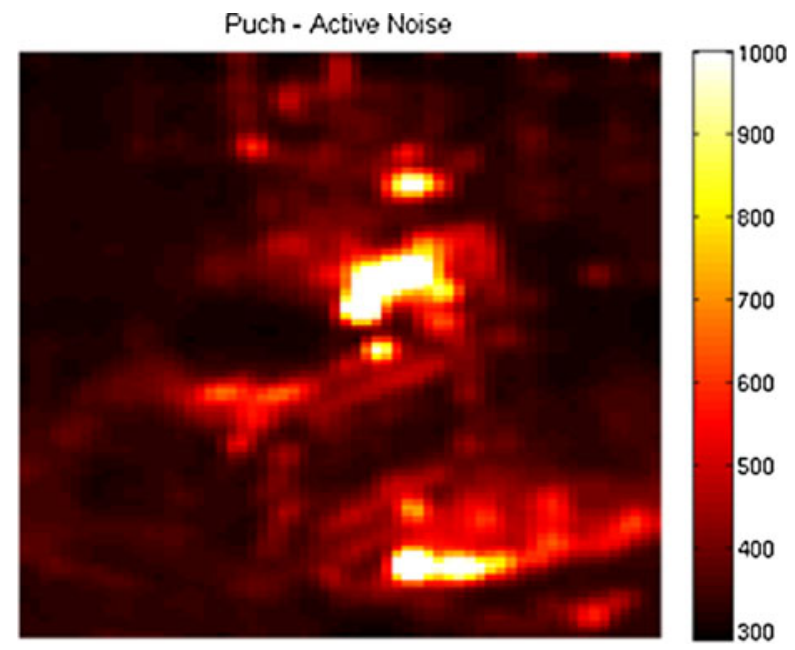

Fig. 19 Active noise image from SPIRA of the Puch vehicle, in brightness temperature [K]

instrument (when the source is fixed) in order to quantify the angular diversity of the setup, as will be later discussed.

The pixel intervals in the PSD decrease moving away from the center to the borders, until reaching the external values which refer to single pixel variations. Through normalization of each PSD, it is possible to compare images which have different dynamic ranges. By comparing the PSD functions of the PUCH vehicle and camouflage nets it is possible to extract additional information about the corresponding active and passive images in term of noise and contrast. This is shown in Figs. from 17 to 26, where the power spectral densities are represented on a logarithmic scale and are normalized over the average power, in order to achieve a better qualitative comparison.

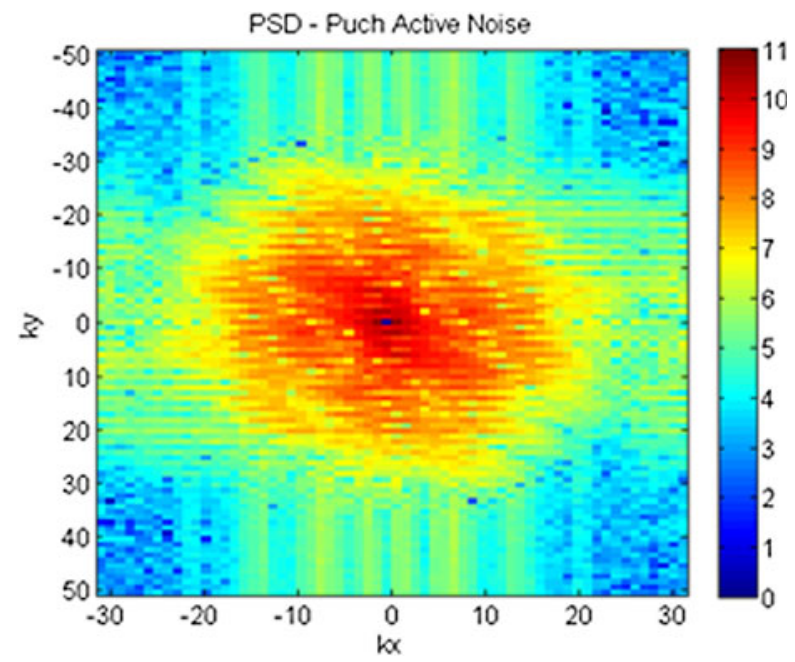

Fig. 20 PSD of the active noise image of the Puch (Fig. 19) in logarithmic scale [dB] 


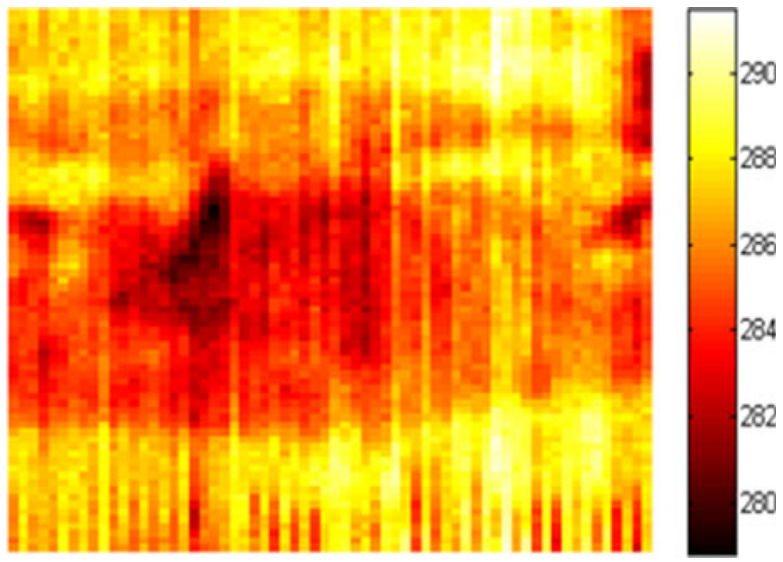

Fig. 21 Passive image of a camouflage net, in brightness temperature [K]

First, we compare the passive and active noise PSDs of the Puch (from Fig. 17 to 20). The diagonal patterns in both PSD functions are directly related to the repetition patterns in the radiometric images. This is clear observing Figs. 17 and 18. The four colder lines in the passive image from bottom left to top right (bumper, hood, windscreen border and roof) are resolved in the PSD in a same amount of peaks from top left to bottom right. This is a well known phenomenon in the 2D Fourier transformation of images, which shows the transitions in the contrast of the images. The more spacing between these peaks in the PSD, the closer this repetition occurs in the image. The wider the peaks, the more is the definition of them in the image (a discrete transition in the image determines an infinite sequence of frequencies to represent it in Fourier domain). The same, in Fig. 17, it is valid for the two main cold edges on the side and on the bottom of the right flank of the car, resulting in a broadening of the PSD (Fig. 18) from bottom left to upper right. Generally the presence of these patterns in the PSD tells us the overall amount of details and contrast in the images.

In Figs. 19 and 20, patterns from different parts of the image are present, resulting in a broader PSD compared to the passive one. The extension among the axes and the intensity of

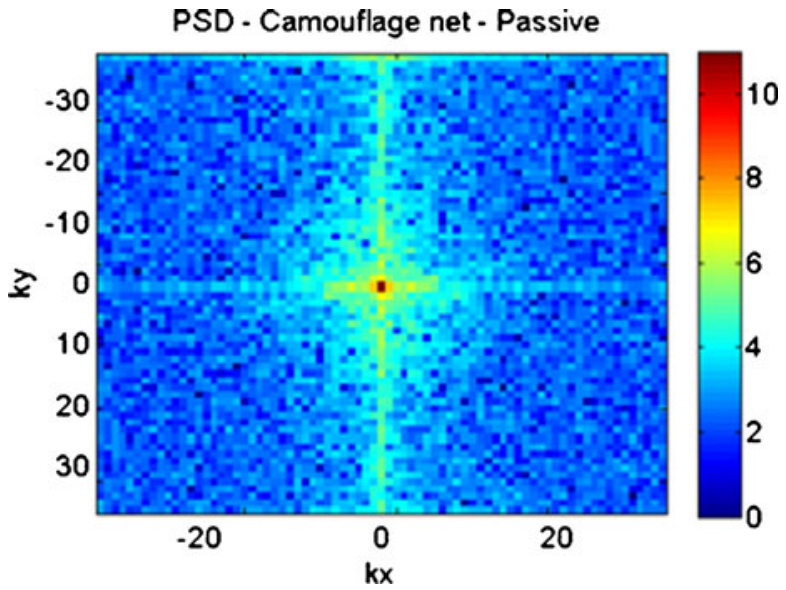

Fig. 22 PSD of passive image of camouflage net (Fig. 21) in logarithmic scale [dB] 


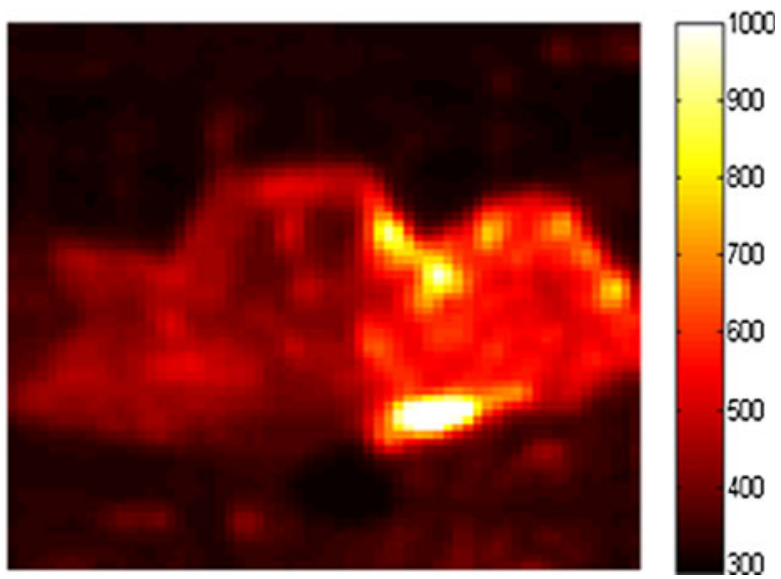

Fig. 23 Active noise illumination image of a camouflage net, in brightness temperature [K]

these patterns in the PSD is inversely proportional to the level of blurring of each detail in the image. In fact, sharp edges require higher spatial frequencies.

Comparing the $-3 \mathrm{~dB}$ circumference in the two PSDs (in our case ellipses, due to the different resolution in $\mathrm{x}$ and $\mathrm{y}$ directions of the images), we can quantitatively compare the overall amount of details in the passive and active images. In our case, the radiuses for passive and active images are:

$$
\begin{aligned}
& \mathrm{Kx}(-3 \mathrm{~dB}), \text { passive } \approx 15 \operatorname{pixel}^{-1} ; \mathrm{Ky}(-3 \mathrm{~dB}), \text { passive } \approx 20 \mathrm{pixel}^{-1} \\
& \mathrm{Kx}(-3 \mathrm{~dB}), \text { active } \approx 22 \operatorname{pixel}^{-1} ; \mathrm{Ky}(-3 \mathrm{~dB}), \text { active } \approx 30 \text { pixel }^{-1}
\end{aligned}
$$

This corresponds to an increase of the $50 \%$ in details for both azimuth and elevation in the noise illumination image. The lines parallel to the two axes which extend up to the border of the PSD are common artifacts due to the fact that many edges in both images are diagonallyoriented. The reason is that the Fourier transformation always treats images as they are part of periodically replicated arrays of identical images extending toward infinity in horizontal and vertical directions. Then, in the contour of adjacent identical images, strong edges are present,

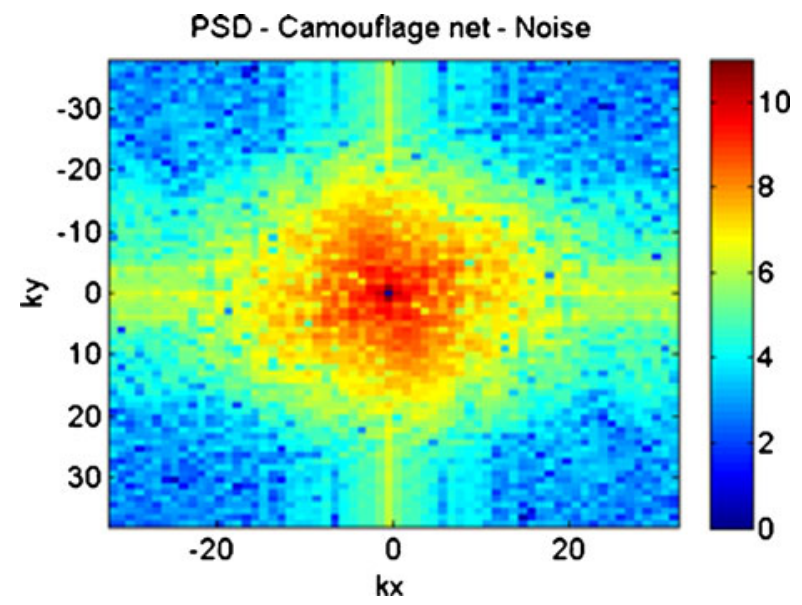

Fig. 24 PSD of active noise image of camouflage net (Fig. 23) in logarithmic scale [dB] 


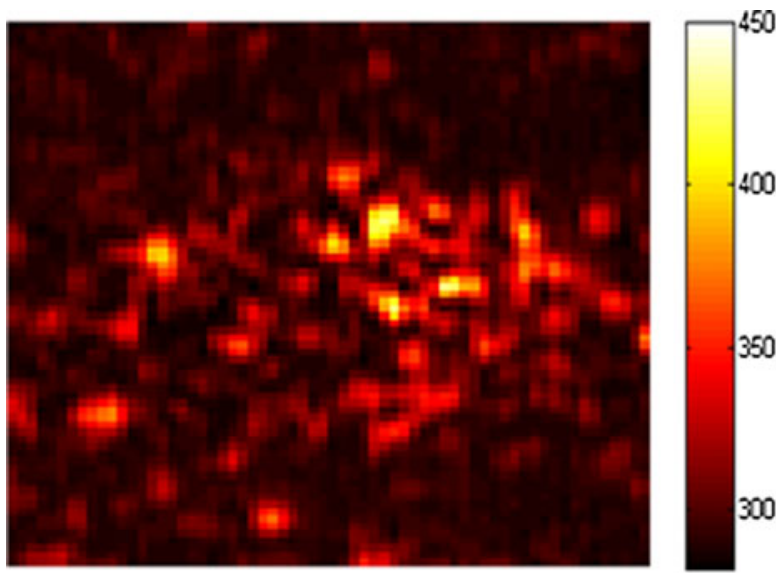

Fig. 25 Active CW illumination image of a camouflage net, in brightness temperature [K]

resulting in high spatial frequency components in the power spectral density. In order to reduce such artifacts, one can dump the external part of the image using a windowing function.

This approach, however, also reduces the details in the real image, contained in high frequency components. In the PSD of passive image (Fig. 18) this "cross" has a stronger component in the horizontal direction, due to the lower correlation among adjacent azimuth lines in SPIRA images, as already reported in literature [13].

In order to summarize the previous considerations, one can assume that the PSD of a well contrasted image has the following characteristics:

- Multiple overlapping periodical patterns centered in $(0,0)$, at different trajectories

- Wide $-3 \mathrm{~dB}$ circumference

- Absence of strong high frequency components in only one direction

The next step is comparing the PSD of noise, CW and passive images of a camouflage net (Fig. 21 to 26). The differences among the three power spectral densities are consistent. The purely passive image (Fig. 21) has a very low contrast; the main power is concentrated only in

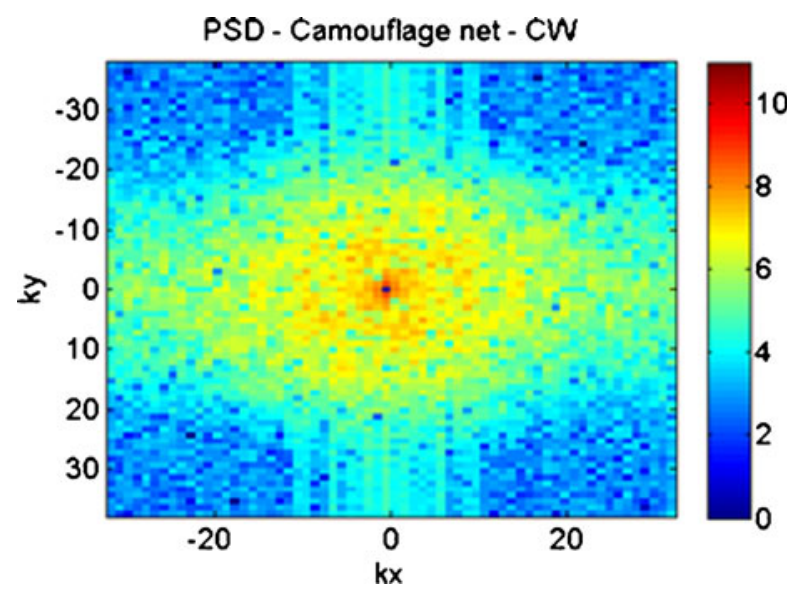

Fig. 26 PSD of active CW image of camouflage net (Fig. 25) in logarithmic scale [dB] 


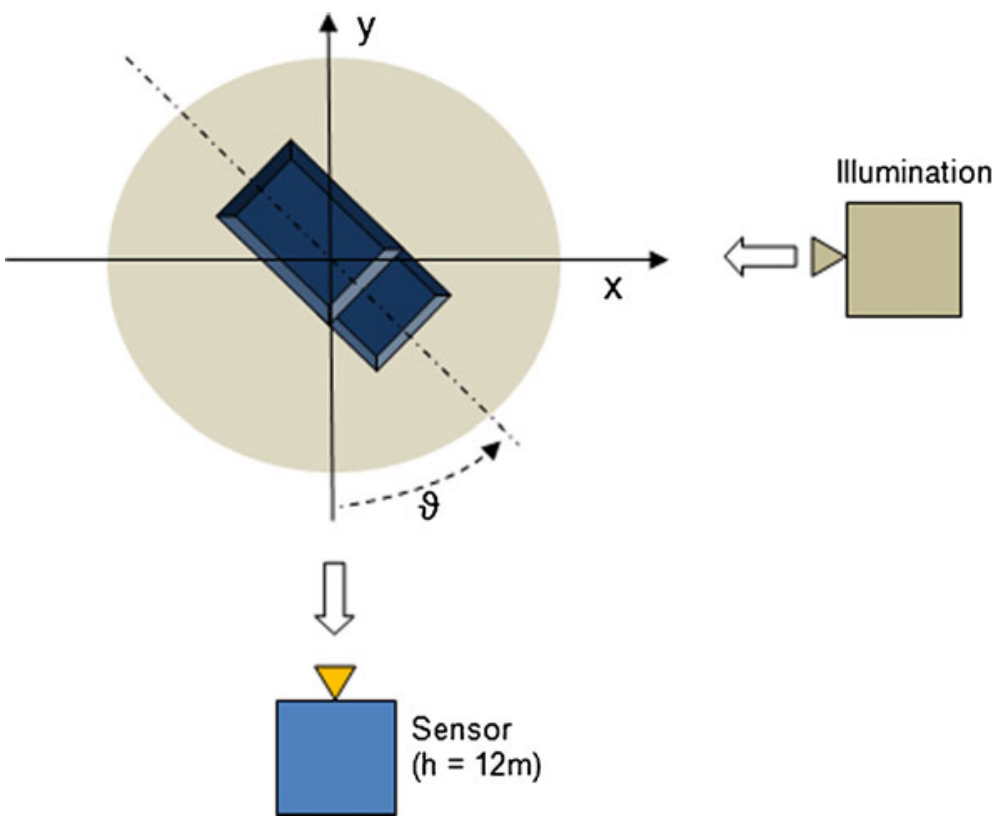

Fig. 27 Test scheme (top view) of the setup for angular diversity measurement. The sensor is placed at a height of 12 meters, along the $y$ axis

the lower spatial frequencies as it is shown in Fig. 22 and Gaussian noise due to the low SNR ratio of indoor passive is observable. In the Fourier domain, in fact, the transformation of white Gaussian noise is a delta in the origin. It is interesting to notice again the presence of a strong line along the horizontal axes in the passive PSD, directly related to the horizontal "colored" noise (period 1 pixel) in the corresponding SPIRA passive image.

Comparing the normalized PSDs of the same target (camouflage net) under noise (Fig. 23) and CW illumination (Fig. 25), it is interesting to notice that a correlation between the two in the high frequency components exists. The considerably higher and broader power spectrum around the origin in the noise PSD (Fig. 24) reveals the overlapping of details of the general structures of the target, while in the PSD of CW illumination (Fig. 26) the power distribution is spread over a wider range of spatial frequencies, up to a corresponding pixel interval of 3 , which is the average radius of the speckle noise. The total power is lower than in the active noise image, due to the statistical properties of speckle noise. In fact, low intensity pixels have the most occurring probability in random path summation, the phenomenon which generates the speckles [15].

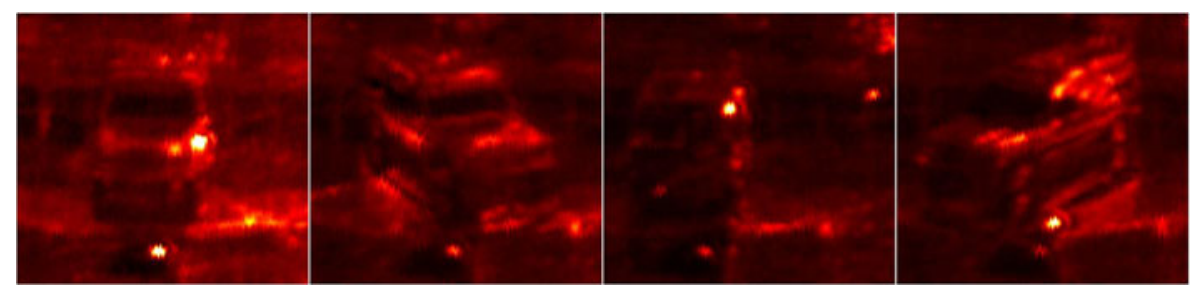

Fig. 28 Active noise illumination images of the Puch vehicle at different angles $\vartheta$ taken by Rotrad 94 


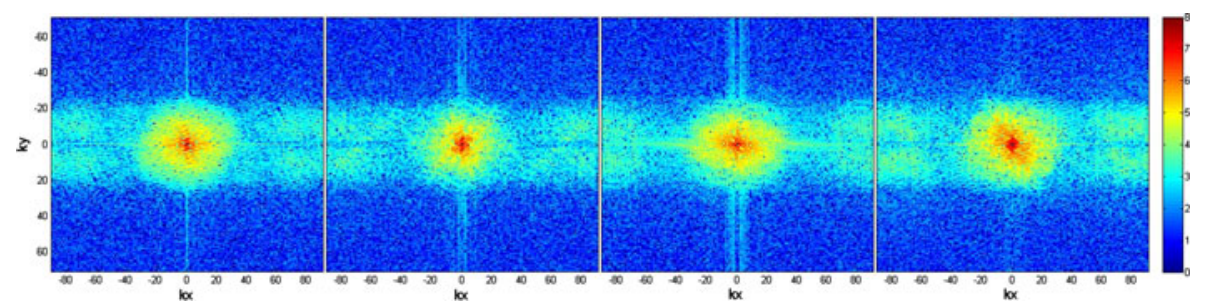

Fig. 29 Corresponding PSDs of the images in Fig. 28, in logarithmic scale [dB]

Theoretically it is possible to filter the PSD in order to isolate as much as possible the spatial frequency components related to the speckle noise, in order to study it and possibly improve the quality of the images, as it is done in laser applications [13].

In conclusion, the analysis of the radiometric images through their power spectral density proved to be a useful tool to better understand the impact of active illumination compared to passive radiometry and generally to analyze the contrast in the images.

\section{Angular Diversity}

Standard monostatic or bistatic illumination systems for active radiometry tend to be strictly dependent on the relative angles between illuminators, target and sensor. Another key factor in such systems is the radiation pattern diameter of the illuminator relative to the target size, in case of static illumination, or the correct alignment of transmitter and receiver in case of tracking illumination.

One of the goals of our experiment was to diffuse the radiation as much as possible around the target, using a wide beam static source together with high reflective walls in order to overcome the limitations of standard illumination methods. Our noise source illuminated the target (Puch car), placed on a rotating platform, from the right side, pointing at the roof above, while the sensors (SPIRA and Rotrad94) were in front of the target at a height of 12 meters, as

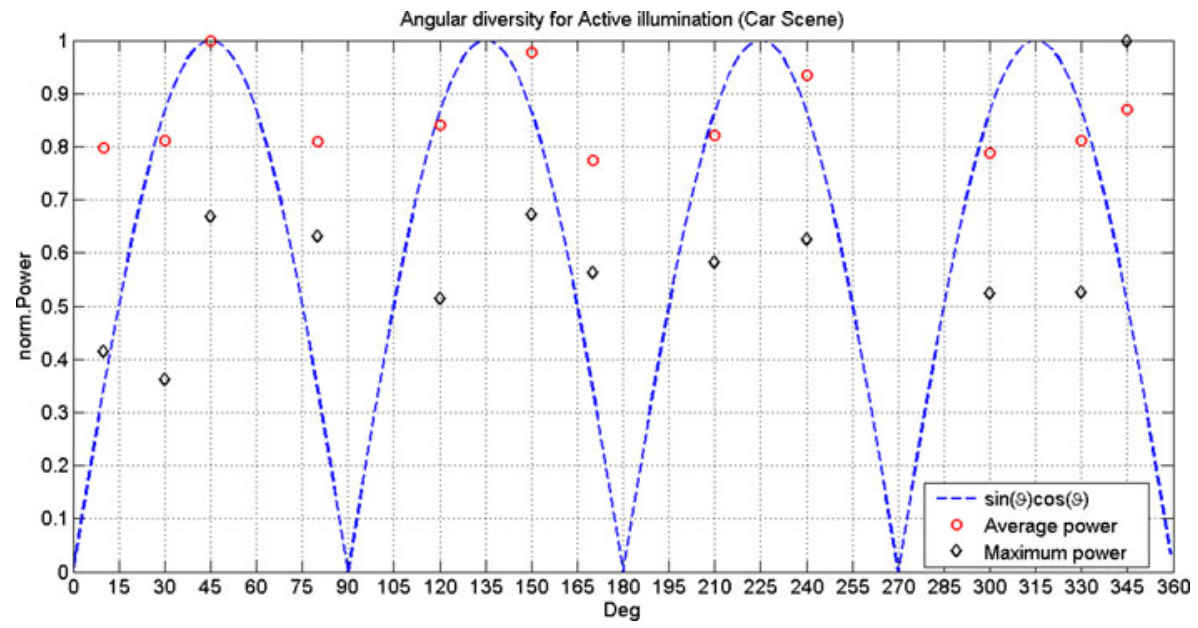

Fig. 30 Angular diversity for active noise illuminated car scene. Normalized average powers and power peak plotted vs. theta angle for each realization 
shown in the following figure. The angle $\vartheta$ is defined as the angle between the longitudinal axis of the Puch and the y-axis (observation direction of the sensor).

The purpose of this test was to acquire images of the Puch car at different $\vartheta$ angles, analyzing the $\vartheta$-dependence of the average power collected by the sensor (Rotrad94 in our setup) in comparison with theoretical expectations (Eq. from 6 to 10, section 3). Due to the fact that Rotrad94 is a fast scanning, uncalibrated radiometer, the images acquired had to be normalized over their maximum pixel values. We obtained a set of images at different angles with a good level of contrast and distributed illumination among the target as shown in Figure 28.

For each image, the PSD has been computed. The power in each orientation is distributed along the same spatial frequencies width, but the orientation of the peaks (PSD levels between 8 and $6 \mathrm{~dB}$ ) reflects the orientation of the target (Fig 29).

The normalized mean power level of each orientation and the peak values were then plotted, in order to show the $\vartheta$-angle dependency of the received power. Considering a Lambertian scatterer as the theoretically ideal case for our setup, like a Puch-size metal cube with diffusely reflective surfaces placed in the center of the platform and illuminated in direct view by the source, the received power $P_{R}$ over $\vartheta$ angles, derived from Eq. 10 in section 3, is proportional to:

$$
P_{R} \propto \sin (\vartheta) \cos (\vartheta)=\frac{1}{2} \sin (2 \vartheta)
$$

With a maxima for $\vartheta=45^{\circ}, 135^{\circ}, 225^{\circ}, 315^{\circ}$. Comparing this case with our experimental result, we can determine how much the average power is following this angular dependency. The more the average power remains constant at different $\vartheta$ angles the more the orientation of the target is not a critical factor thus our active setup realizes a well distributed illumination (similar to cold sky radiation). The following plot shows the result of our computation.

Although a dependency over the $\vartheta$ angle is observable (the power maximums are located around the predicted angles $45^{\circ}, 135^{\circ} \ldots$ ), the average power does not fall under $75 \%$ from the maximum (at $\vartheta=45^{\circ}$ ) in all the other realizations. In other terms, we consistently reduced the angular diversity for active illumination.

This result leads to the conclusion that our diffused noise illumination solution represents an improvement over the standard direct monostatic setups, at the condition than a good distributed illumination on the target is achieved.

\section{Conclusions}

We have successfully verified the application of active noise source illumination to a variety of test scenes in an indoor environment. The results of these test cases show significant improvement in terms of detail and contrast of the observed scenes in comparison to CW source techniques and proved to be an efficient method to increase contrast and detail in indoor radiometric applications. The radiometers used during the campaign were not specifically designed to operate as active illumination detectors, due to their suboptimal dynamic ranges, but nonetheless they proved to be more than sufficient for the task. The use of digital receiver backends could potentially be a major improvement for active illumination radiometry, due to the higher achievable dynamic range.

It should be noted that the experimental environment in which these tests were conducted (hangar with metal wall and roof) was a key factor to the success of the results presented in this paper, providing multiple reflections over the target and confining the illumination within the 
hall. The periodic metal structures of the hall, however, constitute a sub-optimal solution due to the fact that reflections occur only in the three main directions normal to the structure's surfaces. Ideally, a hall made of diffusely reflecting surfaces (Lambertian surfaces) constitutes the optimal case in order to uniformly illuminate the observed target.

Further investigation in more realistic environments will be required to determine the overall efficiency of the proposed technique. We expect that, based on the findings of this work, future test campaigns with more specifically designed radiometers in terms of dynamic range and acquisition speed will yield even better performance.

Acknowledgments The SPIRA instrument and the test campaign have been funded by armasuisse $\mathrm{W}+\mathrm{T}$ under Project No. R-3210/040-11. Special thanks to Dr. Hans Pratisto, Roland Oechslin (armasuisse), Frank Klöppel and Rudolf Schönewald (Fraunofer - FHR) for their precious help during the campaign.

\section{References}

1. N.A.Salmon and R. Appleby, "Sky radiation Temperature changes and fluctuations in the millimeter-wave band", Passive Millimeter- Wave Imaging Technology IV, Proc.SPIE 4032, pp.: 98-102, 2000.

2. E.Heinz et al, "Development of passive submillimeter-wave video imaging systems for security applications", Millimeter Wave and Terahertz sensors and Technology V, Proc. SPIE 8544, 2012.

3. D. Petkie et al, "Active and Passive imaging in the $\mathrm{THz}$ spectral region: phenomenology, dynamic range, modes, and illumination", Journal of Optic Society vol.: 25, no.:9, pp.: 1523-1531, 2008.

4. A. Duric, A. Magun, A. Murk, C. Mätzler, N. Kämpfer, "The Fully Polarimetric Imaging Radiometer SPIRA at $91 \mathrm{GHz}$ ", IEEE Transactions on Geoscience and Remote Sensing, vol.: 46, no.: 8, pp.: 2323-2336, 2008.

5. C.Eiachi, J. Van Zyl, "Introduction to the physics and techniques of Remote Sensing", 2nd Ed. Wiley.

6. A. Murk et al, "Polarimetric Imaging with the $91 \mathrm{GHz}$ Radiometer SPIRA", Millimeter Wave and Terahertz sensors and Technology III, Proc.SPIE 7837, 2010.

7. Duric, A. Magun, "Polarimetric Measurements with a Fully Polarimetric Imaging Radiometer at 91 GHz", Proceedings of International Joint conference of $4^{\text {th }}$ ESA workshop on Millimeter-Wave Technology and App.,Espoo, Finland, February 2006.

8. C. Mätzler, A. Murk, "Active Beleuchtung für Szenen von SPIRA: Vorabklärungen”, Research Report $N^{\circ}$ 2009-11-MW, IAP University of Bern, December 2009.

9. M. Canavero, A. Murk, "Scanning Polarimetric Imaging Radiometer: Microwave Imaging System and Image Merging with IR and Optical Data", Proc. $6^{\text {th }}$ Future Security Research Conference, September 2011.

10. M. Canavero, A. Murk, "Polarimetric Modeling, Calibration and Active Imaging at $91 \mathrm{GHz}$ ", Proc. $6^{\text {th }}$ ESA Workshop on Millimeter Wave Technology and Applications, Espoo, Finland, May 2011.

11. D. Ong et al, "Analysis of laser speckle severity, granularity and anisotropy using the power spectral density in polar coordinates representation”, SPIE Optical Engineering 51(5), May 2012.

12. F. Sarto et al, "Electrode surface morphology characterization by atomic force microscopy", Proc. ICCF-14 International Conf. on Condensed Matter Nuclear Science, vol.: 2, pp.: 437-443, 2008

13. S. Siegenthaler, M. Canavero, A. Murk, "Post Processing of MMW images of SPIRA", Research Report No 3-2011. IAP University of Bern, January 2012.

14. Merril Skolnik, "Introduction to radar systems", second edition, McGraw Hill, 1981.

15. W. Lauterbom, T.Kurz, M. Wiesenfeldt, "Coherent Optics: Fundamentals and Applications", Springer.

16. J.I. Glaser, "Some results in the bistatic radar cross section (RCS) of complex objects", Proceedings of IEEE, vol.: 77 Issue 5, 1989.

17. C. Mätzler, "Applications of the Interaction of the Microwaves with the Natural Snow Cover", Remote Sensing Reviews, vol.: 2, pp.: 259-392, 1987 